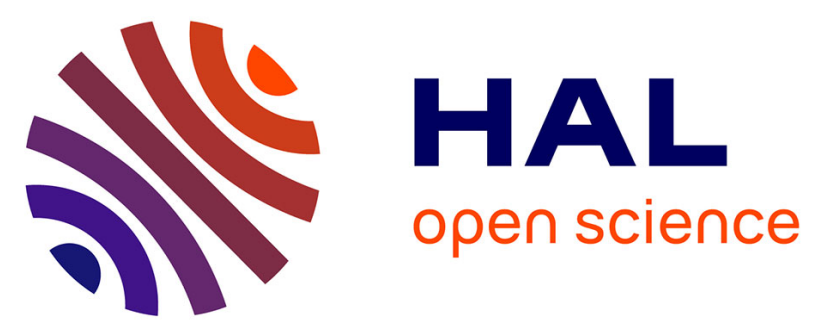

\title{
Back and forth Wolbachia transfers reveal efficient strains to control spotted wing drosophila populations
}

Julien Cattel, Katerina Nikolouli, Thibault Andrieux, Julien Martinez, Francis

Jiggins, Sylvain Charlat, Fabrice Vavre, David Lejon, Patricia Gibert, Laurence Mouton

\section{To cite this version:}

Julien Cattel, Katerina Nikolouli, Thibault Andrieux, Julien Martinez, Francis Jiggins, et al.. Back and forth Wolbachia transfers reveal efficient strains to control spotted wing drosophila populations. Journal of Applied Ecology, 2018, 55 (5), pp.2408-2418. 10.1111/1365-2664.13101 . hal-01916800

\section{HAL Id: hal-01916800 \\ https://hal.science/hal-01916800}

Submitted on 7 Jan 2019

HAL is a multi-disciplinary open access archive for the deposit and dissemination of scientific research documents, whether they are published or not. The documents may come from teaching and research institutions in France or abroad, or from public or private research centers.
L'archive ouverte pluridisciplinaire HAL, est destinée au dépôt et à la diffusion de documents scientifiques de niveau recherche, publiés ou non, émanant des établissements d'enseignement et de recherche français ou étrangers, des laboratoires publics ou privés. 
2 spotted wing drosophila populations

$3 \quad$ J. Cattel ${ }^{1}$; juliencattel@gmail.com

$4 \quad$ K. Nikolouli ${ }^{2,3}$; K.Nikolouli@iaea.org

$5 \quad$ T. Andrieux ${ }^{1}$; thibault.andrieux@ @otmail.fr

$6 \quad$ J. Martinez ; jtm35@cam.ac.uk

$7 \quad$ F. Jiggins ${ }^{4} ;$ fmj1001@ cam.ac.uk

$8 \quad$ S. Charlat ${ }^{1}$; sylvain.charlat@gmail.com

$9 \quad$ F. Vavre ${ }^{1}$; fabrice.vavre@univ-lyon1.fr

10 D. Lejon ${ }^{5}$; dlejon@ rovaltainresearch.com

11 P. Gibert ${ }^{1}$; patricia.gibert@univ-lyon1.fr

12 L. Mouton ${ }^{1}$; laurence.mouton@univ-lyon1.fr

${ }^{1}$ Univ Lyon, Université Claude Bernard, CNRS, Laboratoire de Biométrie et Biologie

Evolutive UMR CNRS 5558, F-69622 Villeurbanne, France

${ }^{2}$ Department of Forest and Soil Sciences, Boku, University of Natural Resources and Life

Sciences, Vienna, Austria

${ }^{3}$ Insect Pest Control Section, Joint FAO/IAEA Division of Nuclear, Techniques in Food and Agriculture, Wagramerstrasse 5, PO Box 100, A-1400 Vienna, Austria

${ }^{4}$ Department of Genetics, University of Cambridge, Cambridge, United Kingdom

${ }^{5}$ Rovaltain Research Company, RRCo, 2 rue René Truhaut, BP10313, 26958 Valence Cedex

Corresponding authors: Julien Cattel, juliencattel@gmail.com, (tel:+33786735362; fax: +33472431388); Laurence Mouton, laurence.mouton@univ-lyon1.fr (tel:+33472432910; fax: $\underline{+33472431388)}$

Key-words: Cytoplasmic Incompatibility, Incompatible Insect Technique, Pest control, spotted wing drosophila, Wolbachia, fruit industry, pesticide, bacteria

Running title: IIT design against $D$. suzukii

Number of words: 7549

Number of tables and figures: 0 table, 6 figures

Supporting information: 4 tables, 5 figures

Number of references: 43 
1. Since its recent invasion of the European and American continents, the spotted wing Drosophila, Drosophila suzukii, has become a burden of the fruit industry. Armed with a highly sclerotized ovipositor, females can lay eggs in a wider variety of ripening and healthy fruits than other Drosophila species. Economic losses due to Drosophila suzukii reach millions of dollars annually and methods to control natural populations in the field mainly rely on the use of chemical pesticides.

2. We tested if Wolbachia bacteria represents a potential ally to control this pest. These symbionts are naturally present in many insects and often induce a form of conditional sterility called Cytoplasmic Incompatibility (CI): the offspring of infected males die, unless the eggs are rescued by the compatible infection, inherited from the mother that protects the embryo. A long-recognised, a strategy called the Incompatible Insect Technique (IIT) makes use of the CI phenotype to control insect populations through the mass release of infected males. To implement this technique in $D$. suzukii, we used back and forth Wolbachia transfers between $D$. suzukii and $D$. simulans to identify Wolbachia strains that can sterilize $D$. suzukii females despite the presence of $w$ Suz, a natural Wolbachia infection in this species.

3. We identified two Wolbachia strains as potential candidates for developing IIT in $D$. suzukii. Both induce a very high level of $\mathrm{CI}$ in this pest which is not attenuated by the presence of $w$ Suz in females. Moreover, the newly transferred Wolbachia do not affect the fitness or the mating competitiveness of the sterilizing males.

4. Synthesis and applications. Although several critical steps still need to be tested and developed outside the laboratory to achieve the control of Drosophila suzukii using Incompatible Insect Technique. By an experimental approach in large population cage, we showed that releases of transinfected males limits population size. Thus, we provide in this 
study the proof of concept that this technique can be a very promising approach to control $D$. suzukii populations.

\section{Introduction}

Drosophila suzukii, the spotted wing Drosophila, has become a major burden for fruit growers since its recent invasion of the European and American continents (Goodhue et al. 2011; Calabria et al. 2012; Cini, Ioriatti \& Anfora 2012; Cini et al. 2014; Asplen et al. 2015). Although the vast majority of Drosophila species are not fruit pests, D. suzukii is able to lay eggs on a wide variety of healthy ripening fruits, thanks to a sclerotized ovipositor (Mitsui, Takahashi \& Kimura 2006). Internal larval feeding represents a direct damage that can also facilitate secondary infections by pathogens as fungi, yeasts or bacteria (Cini, Ioriatti \& Anfora 2012; Hamby et al. 2012; Ioriatti et al. 2015). Most damages are reported on red fruits, with an approximate $\$ 500$ million annual loss in the US (Goodhue et al. 2011). In France and in Northern Italy, up to $100 \%$ destruction was reported on cranberries, strawberries and sweet cherries (Cini, Ioriatti \& Anfora 2012). Control of D. suzukii populations in the field largely relies on chemical pesticides, a practice with serious drawbacks because of its use close to harvest and the consequent risk of high amount of residues left on fruits. In brief, there is an urgent need for developing effective, specific, and environmentally-friendly methods to fight against $D$. suzukii.

In this context, suppression of pest populations through the mass release of sterilizing males seems relevant, as it is highly specific, not polluting, and does not require the introduction of a new species into the environment (Bourtzis et al. 2014; Lees et al. 2015). In the Sterile Insect Technique (SIT), males are sterilized by irradiation and released for mating with wild females (Knipling 1955). This approach has already shown its effectiveness against agricultural insect pests like the New World screw-worm fly (Lindquist, Abusowa \& Hall 
1992). Genetic population suppression approaches, such as Oxitec's RIDL technology could also be considered in principle (Black, Alphey \& James 2011), but this technique faces serious regulatory issues in European Union. Finally, one can make use of the bacterial endosymbiont Wolbachia pipientis to produce sterilizing males (Boller et al. 1976; Riegler \& Stauffer 2002), which is the option explored here.

The genus name "Wolbachia" designates a highly diverse clade of maternally transmitted intracellular symbionts of arthropods and nematodes, belonging to the $\alpha$ proteobacteria (Werren 1997). It is well known for its ability to manipulate host reproduction through different strategies that maximize its spread and maintenance in host populations. Among these, the most common appears to be Cytoplasmic Incompatibility (CI), a sperm-egg incompatibility occurring in crosses between infected males and uninfected females or between males and females infected by incompatible Wolbachia strains, leading to the death of embryos (Werren 1997). CI can be understood as resulting from a modification/rescue system (mod/resc) where the mod function modifies the sperm during spermatogenesis and the resc function, expressed in the egg, rescues the embryo through an interaction with the modified sperm. Recent findings indicate that a pair of genes located in a Wolbachia prophage operon, including a deubiquitylating enzyme, are very likely to encode the mod and resc functions (Beckmann, Ronau \& Hochstrasser 2017; LePage et al. 2017). Depending on Wolbachia and host factors, some strains can both induce and rescue CI, while others express only the resc function, meaning that they do not induce CI but can rescue the embryos against the mod function expressed by other Wolbachia strains (Poinsot et al. 1998). CI has led to the proposal and development of the Incompatible Insect Technique (IIT), analogous to the SIT, where sterilization of the targeted populations is achieved by the release of Wolbachiainfected males incompatible with the resident females (Boller et al. 1976; Riegler \& Stauffer 2002). The first successful application of IIT was achieved in Burma where the target 
population of Culex pipiens, vector of the filariasis, was almost eliminated (Laven 1967). Promising results were also obtained more recently in mosquito species under semi-field (Chambers et al. 2011; Moretti \& Calvitti 2013; Atyame et al. 2015) and field (O'Connor et al. 2012) conditions. While initially limited to species where natural Wolbachia infections allowed the expression of CI, this method came to the fore again within the last decade, thanks to the possibility to transfer Wolbachia strains between species (Hughes \& Rasgon 2014). This approach has been successfully tested in the agricultural pest Ceratitis capitata, naturally uninfected, where the transfer of two Wolbachia strains from Rhagoletis cerasi led to the expression of a high level of CI (Zabalou et al. 2004b).

Here we aim at developing this promising approach in $D$. suzukii, where we face an additional challenge linked to the presence of $w$ Suz, a natural Wolbachia strain, found in most D. suzukii populations but at variable frequencies (Hamm et al. 2014; Cattel et al. 2016a). Data indicated that $w$ Suz is the only natural infection in $D$. suzukii where it does not induce strong CI (Hamm et al. 2014; Cattel et al. 2016a). These results indicate that the implementation of IIT in D. suzukii requires the introduction of a foreign Wolbachia strain that would induce a strong CI in D. suzukii and, crucially, a CI that would not be rescued by $w$ Suz.

To identify such a strain, we first introduced $w$ Suz in Drosophila simulans where many Wolbachia infections are maintained. This allowed us to assess the rescue capacities of $w$ Suz against multiple CI-inducing strains after a single trans-infection experiment. We thus selected three promising strains that were then introduced in D. suzukii leading to the identification of two strains that induce a nearly complete CI in this background, regardless of the presence of $w$ Suz in females. In addition, the transinfected males showed a similar competitiveness compared to naturally infected or uninfected males and are able to induce a high level of CI during all their life. Finally, we demonstrated that in large population cages, 
the IIT can be very efficient to limit the increase of $D$. suzukii populations size. We thus obtained D. suzukii lines combining the properties required for an effective implementation of IIT.

\section{Materials and methods}

\section{MICRO-INJECTIONS AND MATERNAL TRANSMISSION MEASUREMENT}

Micro-injections were performed between D. suzukii and D. simulans, in both directions, using a micro-capillary needle to transfer the cytoplasm of infected embryos into uninfected ones, at the Fly Facility of the Department of Genetics of the University of Cambridge, following Poinsot et al. (1998). Adult females emerging from the injected embryos (G0 females) were crossed with uninfected males of the same genetic background (line STCP in D. simulans and Fr-BE- $\varnothing$ in D. suzukii) and allowed to lay eggs during 5 days. We checked the presence of Wolbachia by PCR (see Table S1 for the protocol) in all G0 females and kept the offspring of the infected ones. This process was repeated until a perfect maternal transmission of Wolbachia was observed. All injected lines were maintained in the lab for at least 8 generations before beginning experiments.

\section{WOLBACHIA STRAINS, DROSOPHILA LINES AND REARING PROCEDURES}

Crossing experiments in D. simulans involved 10 Wolbachia strains, all belonging to the supergroup A (Martinez et al. 2015), 9 of which have been used in earlier studies (Poinsot et al. 1998; Zabalou et al. 2008; Veneti et al. 2012; Martinez et al. 2014) (see Table S2). In $D$. suzukii, we used one isofemale line, named Fr-BE- $\varnothing$, naturally free of Wolbachia, collected in 2012 in Bellegarde (France) and maintained since as a mass population. A line infected by $w$ Suz (Fr-BE-wSuz) was obtained by back-crosses (see Cattel 
et al. 2016b). The Fr-BE- $\varnothing$ and Fr-BE-wSuz lines were maintained for respectively $~ 50$ and 40 generations before the crossing experiments. The Fr-BE- $\varnothing$ line was also the recipient for injections of Wolbachia strains from D. simulans.

D. simulans and D. suzukii lines were reared on a cornmeal diet (agar: $1 \%$, dextrose: $8.75 \%$, maize: $8.75 \%$, yeast: $2 \%$, nipagin: $3 \%$ ) and maintained in an incubator at constant temperature $\left(22^{\circ} \mathrm{C}\right)$ and humidity $(50 \%)$ with a 12 -hours light/dark cycle.

\section{CROSSING EXPERIMENTS}

To analyze the resc function of $w$ Suz in D. simulans we first performed mass crosses using infected females carrying $w$ Suz with males infected by each of the Wolbachia strains available (see Table S2). Freshly emerged adults were sexed and placed separately into cornmeal diet tubes to ensure the virginity of flies. Ten virgin males ( 3 to 5 -days old) and ten virgin females (5 to 6-days old) were allowed to mate in food vials for $24 \mathrm{~h}$. Females were then allowed to oviposit for $48 \mathrm{~h}$ on grape-juice agar in a petri dish. The total number of hatched and unhatched eggs was recorded $48 \mathrm{~h}$ after removal of the females. Six such mass crosses were performed for each Wolbachia strain tested.

Based on the results of mass crosses, three candidate strains were identified $(w \mathrm{Ri}$, $w$ Tei and $w \mathrm{Ha}$ ). To quantify more precisely the CI relationships between $w$ Suz and these candidate Wolbachia strains, individual crosses were performed in D. simulans following the same protocol except that we used only 3-days old virgin males and 5-days old virgin females and that the egg hatch rates were estimated individually.

In $D$. suzukii, in order to make sure that mating had taken place, mating was either observed, confirmed by the hatching of at least one egg, or by the presence of sperm in the spermathecae. We also confirmed $D$. suzukii female's virginity before mating by placing them individually in a petri dish for egg-laying during 48h. Only 13 of the 70 females tested laid 
eggs, but none of them hatched, confirming their virginity. For all individual crosses, at least 20 repetitions were obtained, excluding females that laid fewer than 10 eggs. To account for variation in background embryonic mortality (not related to CI), we used a corrected index of $\mathrm{CI}\left(\mathrm{CI}_{\text {corr }}\right)$ (Poinsot et al. 1998$)$ calculated as follows : $\mathrm{CI}_{\mathrm{corr}}=\left[\left(\mathrm{CI}_{\mathrm{obs}}-\mathrm{CCM}\right) /(100-\mathrm{CCM})\right] *$ 100 , where $\mathrm{CI}_{\mathrm{obs}}$ is the percentage of unhatched eggs observed in a given incompatible cross, and CCM is the mean mortality observed in the control crosses.

\section{MEASURE OF LIFE HISTORY TRAITS}

Wolbachia infection can negatively affect the fitness of its host depending on the Wolbachia strain, the host genotype and the environmental conditions. Because such costs could undermine the effectiveness of the IIT, we measured different life history traits on the lines transinfected by $w \mathrm{Ha}$ and $w$ Tei and compared them to the naturally infected and uninfected lines (Fr-BE- $\varnothing$ and Fr-BE-wSuz). The larval development conditions were standardized in these experiments by depositing 50 eggs of each line in $2 \mathrm{~mL}$ of cornmeal diet placed in a tube with agar and sugar (10\%). We prepared at least 12 such tubes per line and used the freshly emerged adults for the different measures.

We first measured the survival of the pre-adult stages, that is, from egg to adult. In each tube where 50 eggs were deposited, we counted the number of adults that emerged. We then measured the adult survival for both sexes. For each line, 10 freshly emerged adults were placed into a tube containing sweetened $10 \%$ agar (10 replicates per sex) and the mortality was recorded every day. Finally, to measure fecundity and hatch rate, we placed 10 virgin males and 10 females (1-day old) in a cornmeal diet tube during $48 \mathrm{~h}$ for mating. Females were then allowed to oviposit for $48 \mathrm{~h}$ on grape-juice agar in a petri dish. The total number of eggs and their hatch rate were recorded $48 \mathrm{~h}$ after removal of the females. At least 18 repetitions were performed for each line. 


\section{THE EFFET OF MALE AGE ON CI INTENSITY}

To quantify the effect of ageing on CI levels in D. suzukii transinfected lines, we used the same protocol as described above for the mass crosses experiments in D. simulans. The effect of male age on CI intensity was tested for three ages, 3-4, 7-8 or 11-12 days (6 replicates per age), by crossing males infected by $w \mathrm{Ha}$ or $w$ Tei with females naturally infected or not by $w$ Suz. Control crosses were performed (between uninfected males and females, and between males and females carrying $w$ Suz) in order to assess the effect of male age on hatch rate regardless of $\mathrm{CI}$. Here again a corrected $\mathrm{CI}$ index $\left(\mathrm{CI}_{\text {corr }}\right)$ was used in our analysis.

\section{MATING COMPETITIVENESS OF TRANSINFECTED MALES}

The mating competitiveness was tested for males infected by $w \mathrm{Ha}$ by mixing 40 virgin females with different ratios of these sterilizing males with males carrying $w$ Suz or uninfected males in cages of $30 \times 30 \times 30 \mathrm{~cm}$. Five ratios were tested (4 replicates per ratio): 1:1

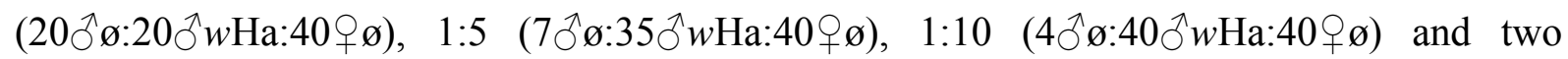

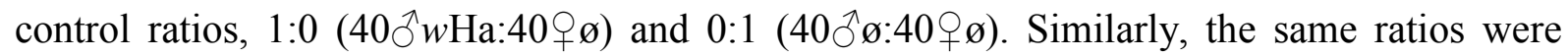
used with males and females carrying $w$ Suz instead of being uninfected. As for previous experiments, we used 5-6 days-old females and 3-4 days-old males. Females were first placed in the cages followed by the simultaneous release of all males, and mating was allowed for 48h, with food and water supply (two recipients containing $50 \mathrm{ml}$ of cornmeal diet, and two sweetened water sources with $10 \%$ sugar). Thereafter, females were allowed to oviposit for 48h on grape-juice agar in petri dishes which were then replaced with new ones for another 48h. The total number of hatched and unhatched eggs was recorded $48 \mathrm{~h}$ after removal the petri dishes from the cage. We computed the competitiveness index (C) (Fried 1971) to compare the performance of sterilizing and compatible males, which is defined as follows: $\mathrm{C}=(\mathrm{N} / \mathrm{S}) *\left[\left(\mathrm{H}_{\mathrm{c}}-\mathrm{H}_{\mathrm{i}}\right) /\left(\mathrm{H}_{\mathrm{i}}-\mathrm{H}_{\mathrm{S}}\right)\right]$, where $\mathrm{N}$ is the number of "compatible" males, $\mathrm{S}$ is the number of 
incompatible males, $\mathrm{H}_{\mathrm{c}}$ is the hatch rate in the compatible crosses, $\mathrm{H}_{\mathrm{i}}$ the hatch rate observed in the different ratios tested and $\mathrm{H}_{\mathrm{s}}$ is the hatch rate in clutches from females exclusively crossed with incompatible males. Similarly, expected hatch rate values in male competition experiments were calculated as follows: $\left[\left(\mathrm{N}^{*} \mathrm{H}_{\mathrm{c}}\right)+\left(\mathrm{S} * \mathrm{H}_{\mathrm{i}}\right)\right] /(\mathrm{S}+\mathrm{N})$.

\section{PROOF OF CONCEPT OF THE IIT EFFECTIVENESS}

The IIT can be used to decrease the population size of the targeted species but also to limit the introduction and the population growth, and this is the point we tested here using males infected by $w$ Ha. Two cages of $3 \times 3 \times 2$ meters were placed separately in climatic chambers with similar conditions of temperature $\left(22^{\circ} \mathrm{C}\right)$, humidity $(50 \%)$, and light (light/dark cycle of 12-hours). In each cage, we placed 6 bottles of $1 \mathrm{~L}$ of a cornmeal diet with a red fruits mixture (red fruits: $50 \%$, agar: $2.6 \%$, yeast: $12 \%$, maize flour: $18 \%$, sugar: $17 \%$, nipagin: $0.4 \%$ ) and 10 bottles of $10 \mathrm{cL}$ of sweetened water $(10 \%)$. In the control cage, 20 4-6 days old mated females and 20 males (half uninfected and half infected by $w$ Suz) were introduced at the beginning of the experiment and then again every 7 days. In the second cage, called the "IIT cage", the same protocol was followed except that in addition to the 40 individuals released every 7 days, we introduced simultaneously 260 sterilizing males, corresponding to a ratio of 13:1 $(260 \hat{w} w \mathrm{Ha}: 20 \hat{\partial}(10 \hat{\varnothing}+10 \widehat{\partial} w \mathrm{Suz}))$. This experiment lasted 62 days, that is, about 9 weeks, so that 360 individuals were released in the control cage and 2700 in the IIT cage (among which 2340 were sterilizing males). The aim of the experiment was to follow the evolution of the population size over time. Six nesting sites (grape-juice agar in a petri dish of $9 \mathrm{~cm}$ ) were placed in each cage and renewed every $48 \mathrm{~h}$. For each nesting site, the number of eggs laid was used as a proxy of the population size and the hatch rate was determined. At the end of the experiment, all living flies were captured and counted. 


\section{STATISTICAL ANALYSIS}

We used generalized linear mixed models (GLMM) (binomial family) to analyse all hatch rates data.

- For the mass and individual crosses in D. simulans and in D. suzukii, the Wolbachia strain was included as a fixed factor and the replicates as a random factor.

- For the mating competitiveness analysis, the factors "ratio" and "female's status" were included as fixed explanatory factors and the replicates as a random factor. Exact binomial tests were then used to compare the observed and expected hatch rates in the mating competitiveness experiment. Survival data were also analyzed by GLMM (gamma distribution and inverse link). The Wolbachia infection was included as a fixed explanatory factor and the replicates vials as a random factor.

- Fecundity data was analyzed using a GLMM (poisson family); the Wolbachia strain was included as a fixed effect and the replicates as a random factor.

- The survival rate from the egg to adult stage was analyzed with a linear mixed-effects model (Gaussian distribution) were the Wolbachia infection was included as fixed explanatory factor and the replicates as a random factor.

- In the last experiment, the number of eggs laid and the hatch rate were analysed using a GLM with a poisson and binomial families respectively.

Analysis were performed in R version 3.3.0 (R Core Team 2016), using the package lme4 for all mixed models (Bates et al. 2014).

\section{Results}

wSuz INJECTION AND CROSSING EXPERIMENTS IN D. SIMULANS

The cytoplasm of $D$. suzukii embryos infected by $w$ Suz was injected into 234 D. simulans Wolbachia-free embryos. Seven isofemale lines proved to be infected by Wolbachia in G0. 
Patterns of maternal transmission of $w \mathrm{Suz}$ are presented in Fig. S1. One isofemale line showing a $100 \%$ transmission from G2 to G3 (20 individuals tested) was selected for CI tests. CI experiments were designed to select potential Wolbachia strains for the sterilization of D. suzukii populations, that is, strains that would induce CI even when females carry $w$ Suz. To this end, we performed crosses in $D$. simulans between males infected by candidate strains and females carrying $w$ Suz. In control crosses, i.e. crosses between males and females both infected by $w$ Suz, the hatch rate was $97.5 \%$. Among the 10 Wolbachia strains tested, known to induce CI in this genetic background (STCP, Martinez et al. 2015), three did not appear to induce CI against $w$ Suz-infected females. Indeed, hatch rates were not significantly reduced in crosses involving the $w$ Stv, $w$ Pro and $w$ MelCS strains compared to the control crosses (Fig. 1). This indicates that $w \operatorname{Suz}$ does express a functional rescue in $D$. simulans against these strains, and discards them as potential candidates. On the contrary, reduced hatch rates were observed in crosses involving males carrying the other strains. Following Poinsot et al. (1998) we computed a $\mathrm{CI}$ index $\left(\mathrm{CI}_{\text {corr }}\right)$ taking into account the basal embryonic mortality, to indicate only the proportion of embryos killed by $\mathrm{CI}$. The highest $\mathrm{CI}_{\text {corr }}$ levels were observed with $w \mathrm{Ri}$ $(57,99 \% \pm 13,73), w \mathrm{Ha}(82,59 \% \pm 11,60)$ and $w$ Tei $(84,74 \% \pm 11,78)$ (Fig. 1).

Focusing on these three promising strains, we performed individual crosses between males carrying these strains and females either uninfected (used as control) or infected with $w$ Suz to characterize more precisely the rescue capabilities of $w$ Suz. We thus showed that the presence of $w$ Suz in females partially rescues the CI induced by $w \mathrm{Ri}$ (with a $29 \%$ decrease of $\left.\mathrm{CI}_{\text {corr }}\right)(z=3.53, \mathrm{P}<0.001)$ and $w$ Tei (with a $23 \%$ decrease in $\left.\mathrm{CI}_{\text {corr }}\right)(z=3.57, \mathrm{P}<0.001)$ but not $w \mathrm{Ha}(z=0.09, \mathrm{P}=0.97)$ (Fig. 2). At that stage, $w \mathrm{Ha}$ thus appeared to be the most promising strain, but we injected all the three strains into D. suzukii in case host effects would change the rescue capabilities of $w$ Suz in its natural host. 


\section{EXPRESSION OF WOLBACHIA CANDIDATES IN D. SUZUKII}

The cytoplasm of $D$. simulans embryos infected by $w \mathrm{Ri}, w \mathrm{Ha}$ or $w$ Tei was injected into uninfected D. suzukii embryos. As for D. simulans, isofemale lines were created from infected females for 2 more other generations until perfect transmission of Wolbachia was observed (from G2 to G3; see details in Table S3). For each Wolbachia strain, one isofemale line was then selected for crossing experiments.

We performed CI crosses to assess (i) if $w \mathrm{Ri}, w \mathrm{Ha}$ and $w$ Tei can induce $\mathrm{CI}$ in $D$. suzukii (transinfected males crossed with uninfected females) and (ii) if $w$ Suz in its natural host is able to rescue these effects (transinfected males crossed with females infected by $w$ Suz). We found that $w \mathrm{Ha}$ and $w$ Tei induce strong CI when infected males are crossed with uninfected females in D. suzukii ( $95.57 \%$ and $96.46 \% \mathrm{CI}_{\text {corr }}$, respectively), in contrast to $w \mathrm{Ri}$ for which the percentage of unhatched eggs was nearly as low as in the control compatible crosses $\left(18.21 \% \mathrm{CI}_{\text {corr }}\right.$ ) (Fig. 3). In addition, the low CI induced by $w$ Ri was fully rescued by the presence of $w$ Suz in females, while the strong CI induced by $w \mathrm{Ha}$ and $w$ Tei was not. When males infected by $w \mathrm{Ha}$ were crossed with females carrying $w$ Suz, only 34 eggs hatched in total out of the 960 eggs laid (3.54\%). Moreover, no egg hatched in 13 of the 30 individual crosses. For the $w$ Tei strain, 33 eggs hatched out of $842(3.92 \%)$ with $0 \%$ hatch rates in 25 of the 33 individual crosses. In two crosses, higher hatch rates (94.7\% and $54.6 \%)$ were seen, suggesting the induction of CI may occasionally fail or that occasionally some males can be uninfected, although an infection rate of $100 \%$ has always been observed in the line used here.

\section{LIFE HISTORY TRAITS OF THE TRANSINFECTED LINES}

Adult longevity data revealed a significant effect of the infection status on this trait in both males and females $\left(\mathrm{P}: \chi^{2}=121.95\right.$, d.f $=3, \mathrm{P}<0.001 ; \delta^{\top}: \chi^{2}=38.98$, d.f $\left.=3, \mathrm{P}<0.001\right)$. However, this effect does not indicate any physiological cost of the $w$ Ha or the $w$ Tei Wolbachia strain. 
On the contrary, males and females infected by $w$ Tei had a greater longevity than the other lines (Fig. S2A and B). In females, there was no significant difference between the three other lines (uninfected, $w$ Suz and $w \mathrm{Ha}$ ) (Fig. S2A) while in males, individuals infected by $w$ Suz showed a higher longevity than uninfected ones (Fig. S2B).

The infection status also affects the fecundity with a significantly larger number of eggs laid in $48 \mathrm{~h}$ in the $w$ Tei $(26.39 \pm 12.03)$ and $w$ Suz lines $(26.22 \pm 8.17)$ (between which no significant difference was found; GLMM: $z=-0.32, \mathrm{P}=0.752$ ). No significant difference was detected between the uninfected $(21.15 \pm 10.82)$ and the $w \mathrm{Ha}(22.29 \pm 9.14)$ lines (GLMM: $z=-$ 0.74, $\mathrm{P}=0.456$ ) (Fig. S3A). We also found an effect of Wolbachia infection on hatch rates, irrespective of CI (Fig. S3B): the $w$ Tei and uninfected lines show higher basal hatch rates $(91.33 \% \pm 15.59$ and $94.59 \% \pm 9.14$ respectively) than the $w \mathrm{Ha}$ and $w$ Suz lines $(83.31 \%$ \pm 25.50 and $83.83 \% \pm 25.36)$.

The infection status also appears to impact survival rates at the pre-adult stage, that is, from egg to adult. Uninfected individuals showed a significantly higher survival rate than the other lines $(\varnothing: 75.33 \% \pm 6.34 ; w$ Suz: $64.38 \% \pm 12.63 ; w$ Tei: $64.00 \% \pm 8.49 ; w$ Ha: $62.17 \%$ \pm 3.86 ), while there was no significant difference between the three infected lines (Fig. S4).

\section{MALE AGE AND CI INTENSITY}

In the control crosses, although hatch rates varied slightly between experiments performed with young or old males, there was no trend indicating an increase or decrease of basal hatch rates with male age (Fig. 4).

In contrast, we observed an overall decrease in CI intensity with male age (Fig. 4A and $\mathrm{B})$. In crosses between males infected by $w \mathrm{Ha}$ and uninfected females, the $\mathrm{CI}_{\text {corr }}$ dropped from $97.65 \%( \pm 2.81)$ in 3-4-days-old males to $79.72 \%( \pm 11.97)$ in 11 -12-days-old males. In 
crosses with $w$ Suz-infected females, the $w \mathrm{Ha} \mathrm{CI}_{\text {corr }}$ dropped from $97.30 \%( \pm 2.50)$ to $78.22 \%$ ( \pm 9.36$)($ Fig. 4A).

The decrease in CI intensity was larger in crosses involving $w$ Tei-infected males, dropping from $94.77 \%( \pm 5.00)$ to $60.68 \%( \pm 16.35)$ and from $93.22 \%( \pm 6.02)$ to $60.71 \%$ $( \pm 10.78)$ in crosses with uninfected and $w$ Suz females, respectively (Fig. 4B). Notably, these experiments also confirmed that females infected by $w$ Suz cannot rescue the CI induced by males infected by $w \mathrm{Ha}$ or $w$ Tei, regardless of male age.

\section{MATING COMPETITIVENESS OF STERILIZING MALES}

In this experiment, we selected the $w$ Ha strain, one among the two candidates, because its CI effect was less affected by male age (full data is provided in Table S4). We first confirmed that $w \mathrm{Ha}$-infected males induced nearly $100 \% \mathrm{CI}$ in $D$. suzukii, regardless of the presence of $w$ Suz in females, with an average hatch rate of $0.40 \%( \pm 1.74)$ and $0.44 \%( \pm 0.56)$ in crosses with uninfected and $w$ Suz females, respectively (Fig. 5A). Accordingly, we observed that hatch rates decrease when the proportion of sterilizing males increases (all ratios tested produce significantly different hatch rates, expect the 1:5 and 1:10 ratios, GLMM: $z=0.63$, $\mathrm{P}=0.60$; Fig. 5A). No effect of females' infection status on hatch rates was detected (GLMM: $z=-0.50, \mathrm{P}=0.62$ ). The hatch rates observed in the ratios 1:0 and 0:1 (without transinfected males or only transinfected males, respectively) allowed us to calculate the hatch rate expected under the assumption of a similar mating competitiveness between sterilizing and compatible males. For each ratio tested, the observed and expected hatch rates were very close, with slight significant deviations. In three cases, the C index (Fried 1971) was less than 1 (meaning that the transinfected males are less competitive than the compatible males) but the reverse was observed in 3 cases (Fig. 5B). Overall, these results indicate that uninfected and $w$ Suz males have very similar mating capacity. 
382 We finally aimed at assessing if repeated releases of sterilizing males could mitigate the increase of a $D$. suzukii population. We performed experiments in two cages of $3 \times 3 \times 2$ meters. In these cages, a small D. suzukii population was introduced (20 mated females and 20 males, half uninfected and half infected by $w$ Suz) and then again every 7 days. In the control cage, where no $w$ Ha-infected males were introduced, the number of eggs laid per time unit (48h) increased substantially during the first 38 days, which corresponds to about two generations (Fig. 6A). The increase continued until the end of the experiment, reaching 1408 eggs laid in 48h. In the IIT cage, where $w$ Ha-infected males were regularly introduced, the number of eggs laid per $48 \mathrm{~h}$ remained low and stable. It was between 0 and 49 per time unit until the 53th days, and slightly increased to reach 308 eggs per time unit at the end of the experiment. The multiple releases of sterilizing males thus allowed to keep the population size 5 times smaller than the control cage and this effect is significant (GLM: $z=-60.74, \mathrm{P}<0.001$ ). Accordingly, the hatch rate observed in the IIT cage was significantly different to the one obtained in the control cage (GLM: $\mathrm{z}=-20.14, \mathrm{P}<0.001)$ (Fig. 6B). Among the 380 eggs laid in the IIT cage in the last 48h, only 120 were viable (39\%) compared with the 1150 hatched eggs among 1408 in the control cage (82\%). At the end of the experiment, that is, after 62 days, all live individuals were caught. We thus counted 2184 individuals in the control cage $(55 \%$ females), and 664 individuals in the IIT cage (41\% females). At the end of the experiment, there were thus 4.3 times less females in the IIT cage than in the control one.

\section{Discussion}

This study aimed at identifying strains of Wolbachia that could be candidates for controlling D. suzukii populations through IIT. We achieved this goal in several steps. We first transferred $w$ Suz, the natural infection of $D$. suzukii, into D. simulans to test its ability to 
rescue the CI induced by the many Wolbachia strains maintained in $D$. simulans. We thereby selected three Wolbachia candidates based on incompatibility with $w$ Suz, injected them into D. suzukii, and validated two as highly promising for the development of IIT.

In the course of these experiments, we confirmed previously described CI patterns, namely a strong host effect affecting compatibility relationships in a strain-specific manner (Reynolds \& Hoffmann 2002; Weeks, Tracy Reynolds \& Hoffmann 2002). We will first discuss these elements before highlighting critical future developments for the effective implementation of IIT in D. suzukii. We found strong variation in CI intensity (mod function) depending on host factors: $w \mathrm{Ri}$ induces only moderate CI in D. suzukii although it is well known to induce strong CI in its natural host D. simulans (Hoffmann, Turelli \& Simmons 1986; this study), but also after transfer in D. melanogaster (Poinsot et al. 1998). On the contrary, wTei does not induce CI in its natural host, D. teissieri (Zabalou et al. 2004a), but induces a high level of CI in both D. suzukii (this study) and D. simulans (Martinez et al. 2015; this study). The $w$ Suz infection exhibits the exact same pattern in its natural host and in D. simulans: it induces very low albeit significant CI (see Fig. S5A, B; 63.7\% hatch rate and $64.2 \%$ respectively). It is clear from these data that a given host should not be generally considered as permissive or refractory to Wolbachia infection and CI, given these strong host genotype-by-Wolbachia strain interactions. Moreover, the analysis of the CI relationship between wSuz and other Wolbachia strains in D. simulans and in D. suzukii reveals that not only the induction of CI (mod function) is host dependent, but also the ability to rescue CI (resc function).

Beyond these CI-relevant results, the main point of this study was to identify Wolbachia strains that could be used as biological control of D. suzukii populations through IIT. By performing back and forth Wolbachia transfers between D. suzukii and D. simulans, we identified two candidates, $w \mathrm{Ha}$ and $w$ Tei. These two strains induce a very high level of CI 
in D. suzukii, which is not attenuated by the presence of $w$ Suz in females. A number of

additional results further confirm that these transinfected lines can be envisaged to implement the control of D. suzukii populations. First, $w \mathrm{Ha}$ and $w$ Tei show a perfect maternal transmission. Second, transinfected males induce a high level of CI throughout their life, despite a reduction with ageing. Finally, transinfected males do not suffer from reduced mating competitiveness or other fitness costs. Accordingly, we showed that the repeated release of sterilizing males can limit the explosion of a $D$. suzukii large cage population. Overall, this study provides, at a laboratory scale, a proof of concept that the IIT approach can be powerful to control D. suzukii populations.

An efficient IIT program must rely on efficient methods to avoid the release of fertile females, which could result in population replacement rather than population suppression (Bourtzis et al. 2014). In case of accidental release, the newly introduced infection would easily spread across $D$. suzukii populations, since the resident infection induces only very low CI. Notably, the fact that the $w$ Ha and $w$ Tei strains are mutually incompatible (Zabalou et al. 2008) means that one strain might still be used for population control in case of accidental release and invasion of the first strain. To circumvent the difficult sexing step, IIT could be coupled with moderate irradiation (Brelsfoard, St Clair \& Dobson 2009; Bourtzis et al. 2014; Zhang et al. 2015) that would be sufficient to sterilize females without affecting the life history traits and competitiveness of males which would be fully sterile thanks to the Wolbachia (Calvitti et al. 2012; Bourtzis et al. 2014). Finally, IIT relies on the massive production of males which seems achievable for such a small and polyphagous insect, but requires additional developments.

The existence of D. suzukii lines transinfected by CI inducing Wolbachia strains represents a critical step toward the implementation of IIT. Our results indicate that these lines carry a number of crucial properties that make them usable in practice to limit population size 


\section{Supporting Information}

478 Table S1. Primers used in this study

\section{Author's contributions} publication.

\section{Acknowledgments} Planche for their technical contribution.

\section{Data accessibility} 2018).

in large population cages. While additional developments are still needed, we are now much closer to make IIT a credible alternative to pesticides to control $D$. suzukii populations.

JC, SC, FV, PG and LM conceived the project and designed its methodology; JC, TA, KN and DL collected the data; JC analyzed the data; JC, SC, FV, PG and LM led the writing of the manuscript. All authors contributed critically to the drafts and gave final approval for

This work was funded by the CNRS (IFR41-UMR5558) and supported by the ONEMA (APR Biodiversité-Ecophyto), the ANR (ANR-15-CE21-0017-01) and the FWF (I 2604-B25). Julien Cattel was the recipient of a $\mathrm{PhD}$ studentship from the Rhône-Alpes region ("ARC Program" Grant). We also warmly thank Yuk Sang Chan at Cambridge University, for his invaluable competence on Wolbachia transinfections, and Aymeric Bellemain and Justine

Data available from the Dryad Digital Repository. DOI: 10.5061/dryad.4c7kq (Cattel et al.
Table S2. Details of all D. simulans lines used in this study 
480 Table S3. Data on microinjections and maternal transmission of Wolbachia strains in D.

481 suzukii

482 Table S4. Data on mating competitiveness of $w$ Ha-transinfected males

483 Figure S1. Details of transmission rate of $w$ Suz in isofemale line of $D$. simulans

484 Figure S2. Effect of Wolbachia infection on D. suzukii survival

485 Figure S3. Effect of Wolbachia infection on the fecundity and the hatch rate in D. suzukii

486 Figure S4. Effect of Wolbachia infection on the survival rate from the egg to adult stage

$487 \quad$ Figure S5. CI induced by $w$ Suz in $D$. suzukii and $D$. simulans 
Asplen, M.K., Anfora, G., Biondi, A., Choi, D.S., Chu, D., Daane, K.M., Gibert, P., Gutierrez, A.P., Hoelmer, K.A., Hutchison, W.D., Isaacs, R., Jiang, Z.L., Kárpáti, Z., Kimura, M.T., Pascual, M., Philips, C.R., Plantamp, C., Ponti, L., Vétek, G., Vogt, H., Walton, V.M., Yu, Y., Zappalà, L. \& Desneux, N. (2015) Invasion biology of spotted wing Drosophila (Drosophila suzukii): a global perspective and future priorities. Journal of Pest Science, 88, 469-494.

Atyame, C.M., Cattel, J., Lebon, C., Flores, O., Dehecq, J.S., Weill, M., Gouagna, L.C. \& Tortosa, P. (2015) Wolbachia-based population control strategy targeting Culex quinquefasciatus mosquitoes proves efficient under semi-field conditions. PLOS ONE, 10, $1-15$.

Bates, D., Maechler, M., Bolker, B., Walker, S. \& others. (2014) lme4: Linear mixed-effects models using Eigen and S4. R package version, 1, 1-23.

Beckmann, J.F., Ronau, J.A. \& Hochstrasser, M. (2017) A Wolbachia deubiquitylating enzyme induces cytoplasmic incompatibility. Nature Microbiology, 2, 17007.

Black, W.C., Alphey, L. \& James, A.A. (2011) Why RIDL is not SIT. Trends in Parasitology, 27, 362-370.

Boller, E.F., Russ, K., Vallo, V. \& Bush, G.L. (1976) Incompatible Races of European Cherry Fruit-Fly, Rhagoletis-Cerasi (Diptera-Tephritidae), Their Origin and Potential Use in Biological-Control. Entomologia Experimentalis et Applicata, 20, 237-247.

Bourtzis, K., Dobson, S.L., Xi, Z., Rasgon, J.L., Calvitti, M., Moreira, L.A., Bossin, H.C., Moretti, R., Baton, L.A., Hughes, G.L., Mavingui, P. \& Gilles, J.R.L. (2014) Harnessing mosquito-Wolbachia symbiosis for vector and disease control. Acta Tropica, 132, S150S163.

Brelsfoard, C.L., St Clair, W. \& Dobson, S.L. (2009) Integration of irradiation with cytoplasmic incompatibility to facilitate a lymphatic filariasis vector elimination approach. Parasites \& vectors, 2, 38.

Calabria, G., Máca, J., Bächli, G., Serra, L. \& Pascual, M. (2012) First records of the potential pest species Drosophila suzukii (Diptera: Drosophilidae) in Europe. Journal of Applied Entomology, 136, 139-147.

Calvitti, M., Moretti, R., Skidmore, A.R. \& Dobson, S.L. (2012) Wolbachia strain wPip yields a pattern of cytoplasmic incompatibility enhancing a Wolbachia-based suppression strategy against the disease vector Aedes albopictus. Parasites \& vectors, 5 , 254.

Cattel, J., Kaur, R., Gibert, P., Martinez, J., Fraimout, A., Jiggins, F., Andrieux, T., Siozios, S., Anfora, G., Miller, W., Rota-Stabelli, O. \& Mouton, L. (2016a) Wolbachia in European populations of the invasive pest Drosophila suzukii: Regional variation in infection frequencies. PLoS ONE, 11, 1-12.

Cattel, J., Martinez, J., Jiggins, F., Mouton, L. \& Gibert, P. (2016b) Wolbachia-mediated protection against viruses in the invasive pest Drosophila suzukii. Insect Molecular Biology, 25, 595-603.

Cattel, J., Nikolouli, K., Andrieux, T., Martinez, J., Jiggins, F., Charlat, S., Vavre, F., Lejon, D., Gibert, P., Mouton, L. (2018) Data from: Back and forth Wolbachia transfers reveal efficient strains to control spotted wing drosophila populations (2018). Dryad Digital Repository. http://dx.doi.org/10.5061/dryad.4c7kq.

Chambers, E.W., Hapairai, L., Peel, B.A., Bossin, H. \& Dobson, S.L. (2011) Male mating competitiveness of a Wolbachia-introgressed Aedes polynesiensis strain under semi-field conditions. PLoS Neglected Tropical Diseases, 5, 1-6.

Cini, A., Anfora, G., Escudero-Colomar, L.A., Grassi, A., Santosuosso, U., Seljak, G. \& 
Papini, A. (2014) Tracking the invasion of the alien fruit pest Drosophila suzukii in Europe. Journal of Pest Science, 87, 559-566.

Cini, A., Ioriatti, C. \& Anfora, G. (2012) A review of the invasion of Drosophila suzukii in Europe and a draft research agenda for integrated pest management. Bulletin of Insectology, 65, 149-160.

Fried, M. (1971) Determination of sterile-insect competitiveness. Journal of Economic Entomology, 64, 869-872.

Goodhue, R.E., Bolda, M., Farnsworth, D., Williams, J.C. \& Zalom, F.G. (2011) Spotted wing drosophila infestation of California strawberries and raspberries: Economic analysis of potential revenue losses and control costs. Pest Management Science, 67, 1396-1402.

Hamby, K.A., Hernández, A., Boundy-Mills, K. \& Zalom, F.G. (2012) Associations of yeasts with spotted-wing Drosophila (Drosophila suzukii; Diptera: Drosophilidae) in cherries and raspberries. Applied and Environmental Microbiology, 78, 4869-4873.

Hamm, C.A., Begun, D.J., Vo, A., Smith, C.C.R., Saelao, P., Shaver, A.O., Jaenike, J. \& Turelli, M. (2014) Wolbachia do not live by reproductive manipulation alone: Infection polymorphism in Drosophila suzukii and D. Subpulchrella. Molecular Ecology, 23, 4871-4885.

Hoffmann, A.A., Turelli, M. \& Simmons, G.M. (1986) Unidirectional incompatibility between populations of Drosophila simulans. Evolution, 692-701.

Hughes, G.L. \& Rasgon, J.L. (2014) Transinfection: A method to investigate Wolbachia-host interactions and control arthropod-borne disease. Insect Molecular Biology, 23, 141-151.

Ioriatti, C., Walton, V., Dalton, D., Anfora, G., Grassi, A., Maistri, S. \& Mazzoni, V. (2015) Drosophila suzukii (Diptera: Drosophilidae) and Its Potential Impact to Wine Grapes During Harvest in Two Cool Climate Wine Grape Production Regions. Journal of Economic Entomology, 108, 1148-1155.

Knipling, E.F. (1955) Possibilities of insect control or eradication through the use of sexually sterile males. Journal of Economic Entomology, 48, 459-462.

Laven, H. (1967) Eradication of Culex pipiens fatigans through cytoplasmic incompatibility. Nature, London, 216, 383-384.

Lees, R.S., Gilles, J.R., Hendrichs, J., Vreysen, M.J. \& Bourtzis, K. (2015) Back to the future: the sterile insect technique against mosquito disease vectors. Current Opinion in Insect Science, 10, 156-162.

LePage, D.P., Metcalf, J.A., Bordenstein, S.R., On, J., Perlmutter, J.I., Shropshire, J.D., Layton, E.M., Funkhouser-Jones, L.J., Beckmann, J.F. \& Bordenstein, S.R. (2017) Prophage WO genes recapitulate and enhance Wolbachia-induced cytoplasmic incompatibility. Nature, 543, 243-247.

Lindquist, D.A., Abusowa, M. \& Hall, M.J.R. (1992) The New World screwworm fly in Libya: a review of its introduction and eradication. Medical and Veterinary Entomology, 6, 2-8.

Martinez, J., Longdon, B., Bauer, S., Chan, Y.S., Miller, W.J., Bourtzis, K., Teixeira, L. \& Jiggins, F.M. (2014) Symbionts Commonly Provide Broad Spectrum Resistance to Viruses in Insects: A Comparative Analysis of Wolbachia Strains. PLoS Pathogens, 10. Martinez, J., Ok, S., Smith, S., Snoeck, K., Day, J.P. \& Jiggins, F.M. (2015) Should Symbionts Be Nice or Selfish? Antiviral Effects of Wolbachia Are Costly but Reproductive Parasitism Is Not. PLoS Pathogens, 11, 1-20.

Mitsui, H., Takahashi, K.H. \& Kimura, M.T. (2006) Spatial distributions and clutch sizes of Drosophila species ovipositing on cherry fruits of different stages. Population Ecology, 48, 233-237.

Moretti, R. \& Calvitti, M. (2013) Male mating performance and cytoplasmic incompatibility 
in a wPip Wolbachia trans-infected line of Aedes albopictus (Stegomyia albopicta). Medical and Veterinary Entomology, 27, 377-386.

O’Connor, L., Plichart, C., Sang, A.C., Brelsfoard, C.L., Bossin, H.C. \& Dobson, S.L. (2012) Open Release of Male Mosquitoes Infected with a Wolbachia Biopesticide: Field Performance and Infection Containment. PLoS Neglected Tropical Diseases, 6.

Poinsot, D., Bourtzis, K., Markakis, G., Savakis, C. \& Merçot, H. (1998) Wolbachia Transfer from Drosophila melanogaster into D. simulans: Host Effect and Cytoplasmic Incompatibility Relationships. Genetics, 150, 227-237.

R Core Team. (2016) R: A language and environment for statistical computing. R Foundation for Statistical Computing, Vienna, Austria. 2016.

Reynolds, K.T. \& Hoffmann, A.A. (2002) Male age, host effects and the weak expression or non-expression of cytoplasmic incompatibility in Drosophila strains infected by maternally transmitted Wolbachia. Genetical research, 80, 79-87.

Riegler, M. \& Stauffer, C. (2002) Wolbachia infections and superinfections in cytoplasmically incompatible populations of the European cherry fruit fly Rhagoletis cerasi (Diptera, Tephritidae). Molecular Ecology, 11, 2425-2434.

Veneti, Z., Zabalou, S., Papafotiou, G., Paraskevopoulos, C., Pattas, S., Livadaras, I., Markakis, G., Herren, J.K., Jaenike, J. \& Bourtzis, K. (2012) Loss of reproductive parasitism following transfer of male-killing Wolbachia to Drosophila melanogaster and Drosophila simulans. Heredity, 109, 306-312.

Weeks, A.R., Tracy Reynolds, K. \& Hoffmann, A.A. (2002) Wolbachia dynamics and host effects: What has (and has not) been demonstrated? Trends in Ecology and Evolution, 17, 257-262.

Werren, J.H. (1997) Biology of Wolbachia. Annu. Rev. Entomol., 124, 587-609.

Zabalou, S., Apostolaki, A., Pattas, S., Veneti, Z., Paraskevopoulos, C., Livadaras, I., Markakis, G., Brissac, T., Merçot, H. \& Bourtzis, K. (2008) Multiple rescue factors within a Wolbachia strain. Genetics, 178, 2145-2160.

Zabalou, S., Charlat, S., Nirgianaki, A., Lachaise, D., Merçot, H. \& Bourtzis, K. (2004a) Natural Wolbachia infections in the Drosophila yakuba species complex do not induce cytoplasmic incompatibility but fully rescue the wRi modification. Genetics, 167, 827834.

Zabalou, S., Riegler, M., Theodorakopoulou, M., Stauffer, C., Savakis, C. \& Bourtzis, K. (2004b) Wolbachia-induced cytoplasmic incompatibility as a means for insect pest population control. Proceedings of the National Academy of Sciences of the United States of America, 101, 15042-15045.

Zhang, D., Zheng, X., Xi, Z., Bourtzis, K. \& Gilles, J.R.L. (2015) Combining the sterile insect technique with the incompatible insect technique: I-impact of Wolbachia infection on the fitness of triple- and double-infected strains of Aedes albopictus. PLOS ONE, 10, 1-13. 
Figures

636

637

638

639

640

641

642

643

644

645

646

647

648 Fig .1. CI levels estimated in mass crosses in D. simulans between infected males (Wolbachia

649 strains named on the horizontal axis) and $w$ Suz-infected females. The $\mathrm{CI}_{\text {corr }}$ index removes the

650 basal embryonic mortality (estimated in control crosses); it is thus a measure of the CI-related 651 mortality. GLMM (binomial family) was performed for comparisons with the control crosses.

$652 * * *: P<0.001 ; *: P<0.05$.

653

654

655

656 


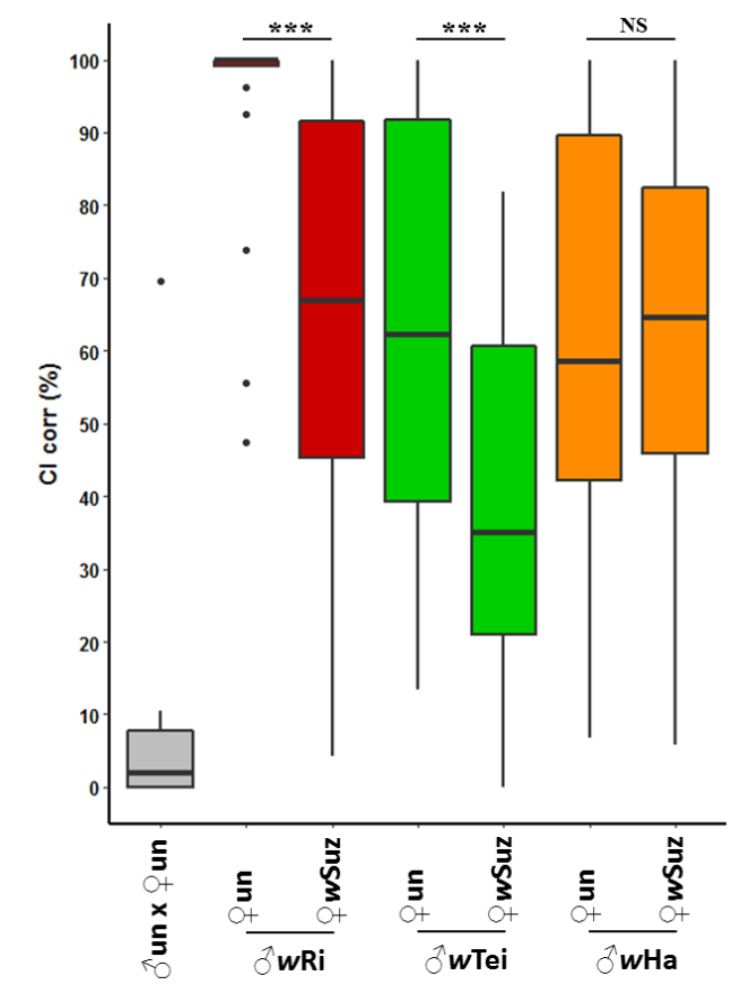

667

668 Fig .2. Assessment of the resc capabilities of $w$ Suz in D. simulans. un: uninfected, $w$ Suz: 669 infected by $w$ Suz, $w$ Ri: infected by $w \mathrm{Ri}, w$ Tei: infected by $w$ Tei, $w$ Ha: infected by $w$ Ha. The $670 \mathrm{CI}_{\text {corr }}$ index removes the basal embryonic mortality (estimated in control crosses); it is thus a 671 measure of the CI-related mortality. 20 repetitions were performed for each type of cross. $672 * * *: P<0.001$.

673

674

675 
680

681

682

683

684

685

686

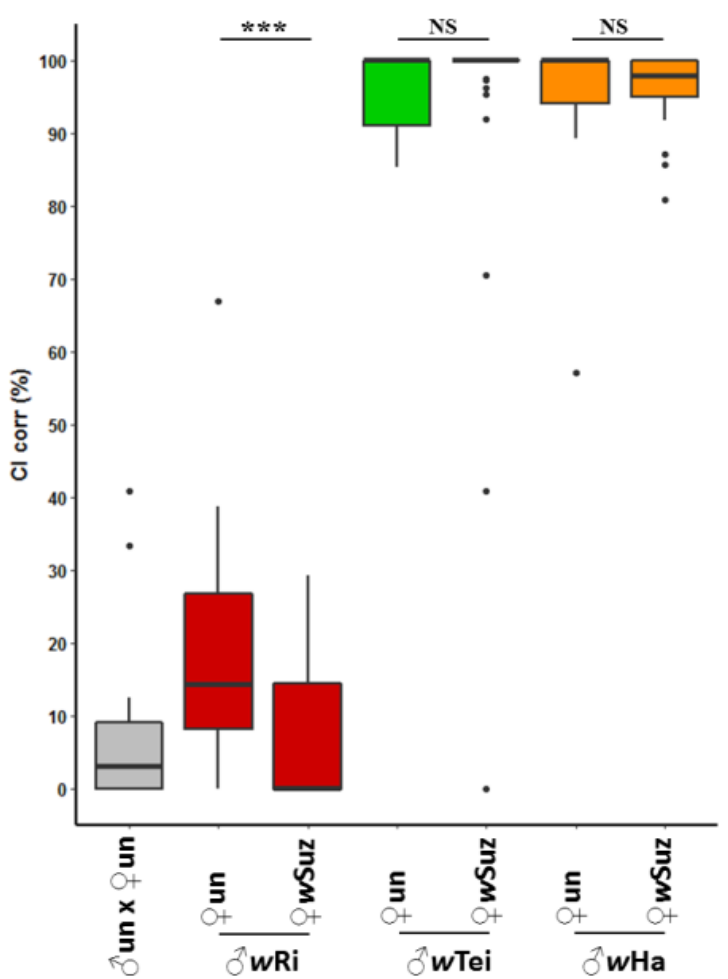

687 Fig .3. Measure of the $\bmod$ function of $w \mathrm{Ri}, w \mathrm{Ha}$ and $w$ Tei and the resc function of $w$ Suz in 688 D. suzukii. un: uninfected, $w$ Suz: infected by $w \mathrm{Suz}, w \mathrm{Ri}$ : infected by $w \mathrm{Ri}, w$ Tei: infected by $689 w$ Tei, $w \mathrm{Ha}$ : infected by $w \mathrm{Ha}$. The $\mathrm{CI}_{\text {corr }}$ index removes the basal embryonic mortality 690 (estimated in control crosses); it is thus a measure of the CI-related mortality. ${ }^{* * *}: P<0.001$.

691

692 


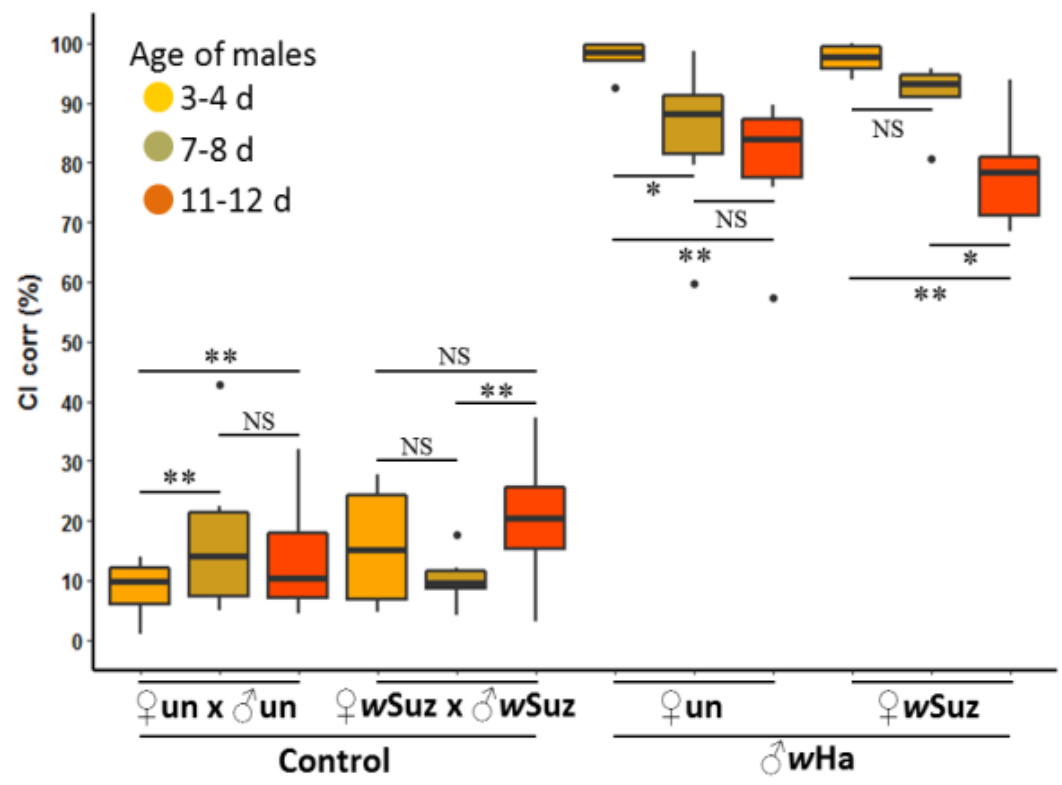

702

B

704

705

706

707

708

709

710

711

712

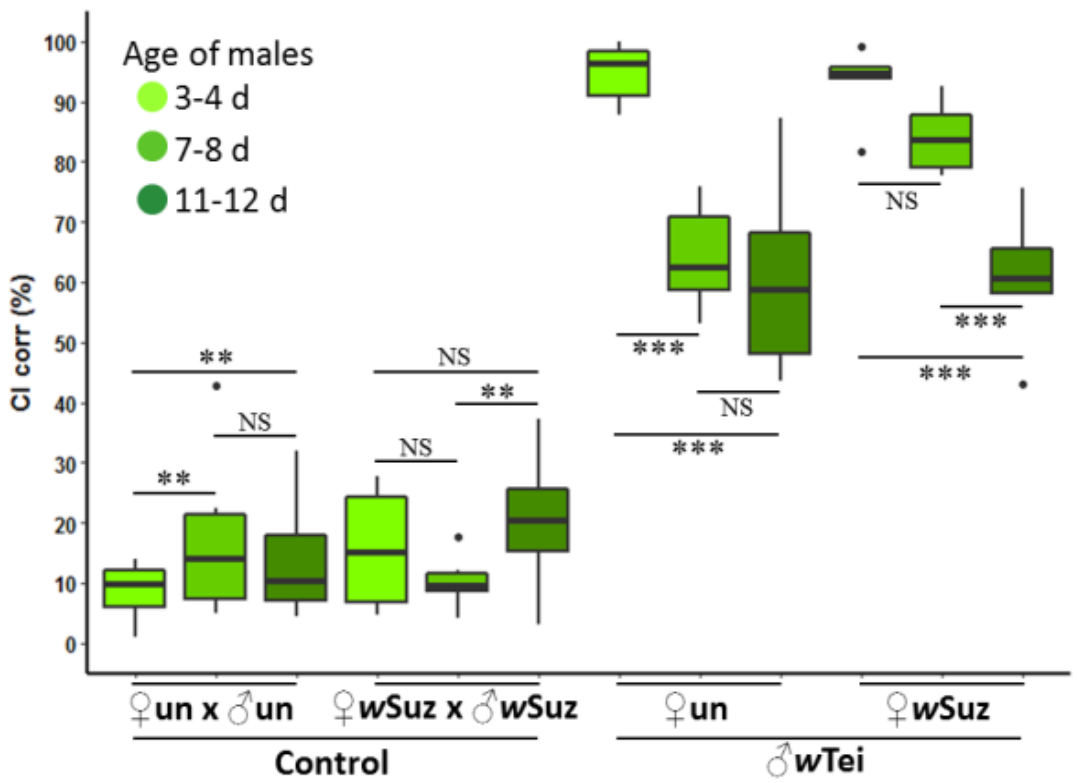

713

Fig. 4. Effect of male age on CI intensity in D. suzukii. For each transinfected line (wHa or $w$ Tei), three different ages were tested, 3-4, 7-8 and 11-12 days. A: sterilizing males infected by $w$ Ha. B: sterilizing males infected by $w$ Tei. un: uninfected, $w$ Suz: infected by $w$ Suz, $w$ Ha: infected by $w \mathrm{Ha}, w$ Tei: infected by $w$ Tei. The $\mathrm{CI}_{\text {corr }}$ index removes the basal embryonic mortality (estimated in control crosses); it is thus a measure of the CI-related mortality. $* * *: \mathrm{P}<0.001, * *: \mathrm{P}<0.01, *: \mathrm{P}<0.05$. 
723

724

725

726

727

728

729

730

731

732

733

734

735

736

737

738

739

740

741

742

743
B

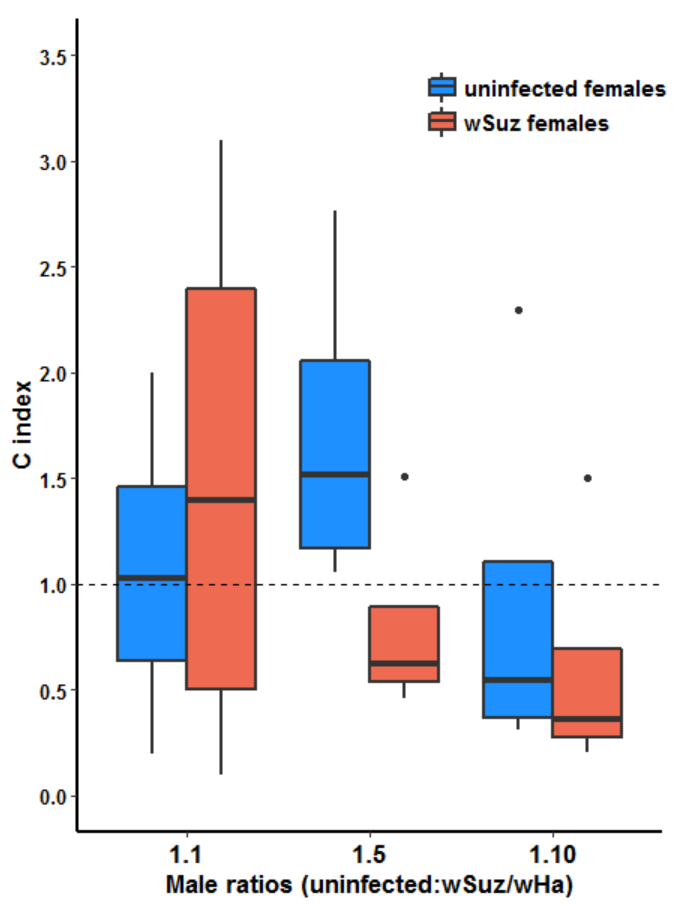

Male ratios (uninfected:wSuz/wHa)

Fig. 5. Mating competitiveness of the $w$ Ha-transinfected males. A: observed and expected hatch rates; as expected, hatch rates decrease as the proportion of sterilizing males is increased. Exact binomial tests were performed to compare the observed and expected hatch values. ***: $\mathrm{P}<0.001$; **: $\mathrm{P}<0.01$. $\mathrm{B}$ : competitiveness index $(\mathrm{C})$. Both expected hatch rates and C index were computed following Fried (1971) (see Material and Methods for details).

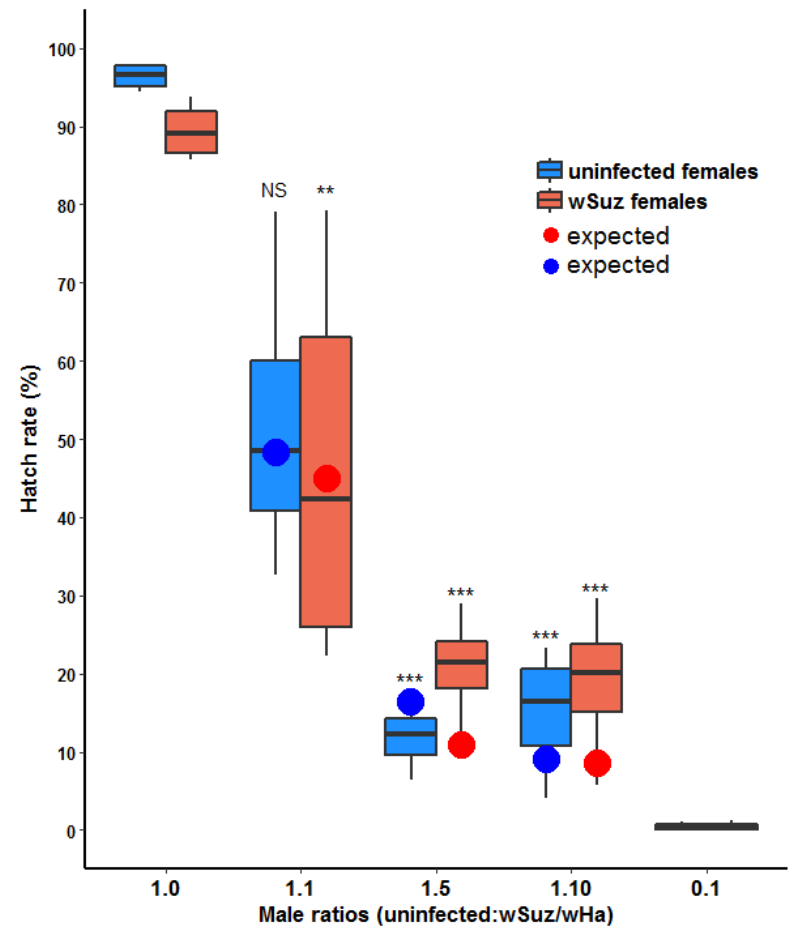


745 A

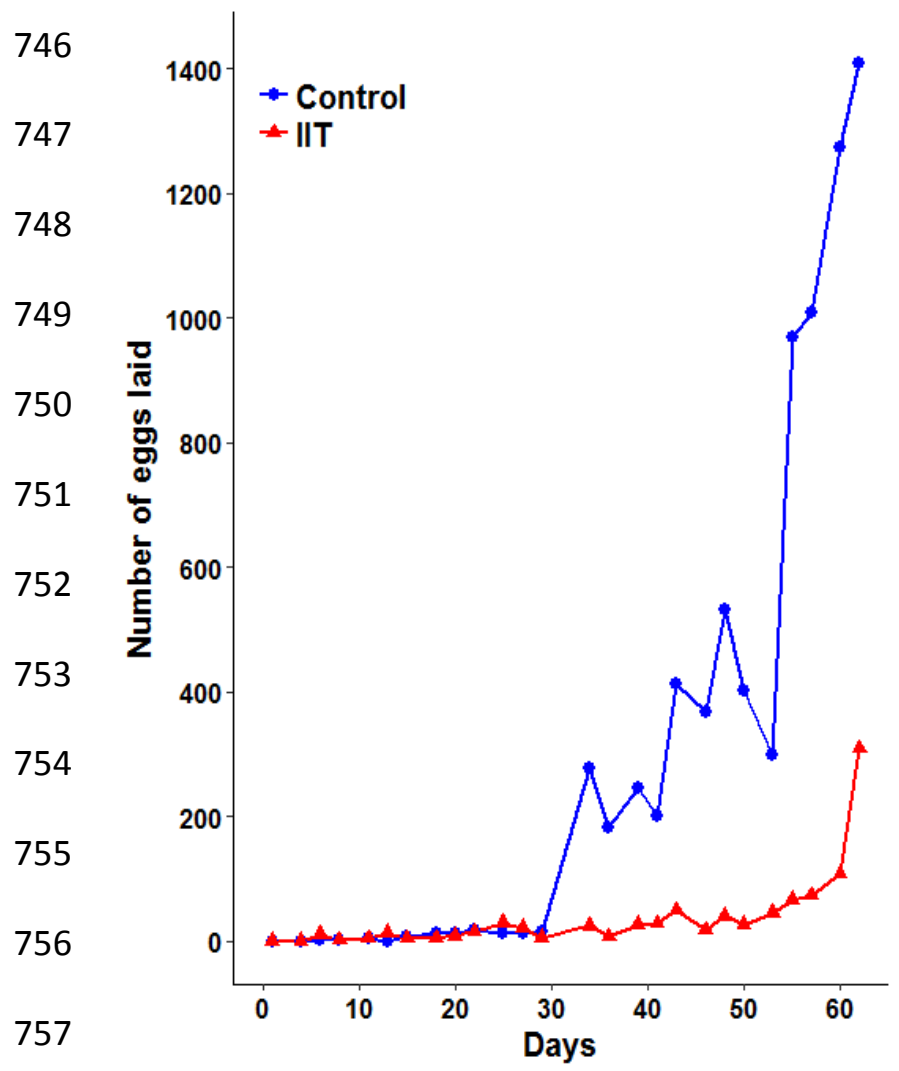

B

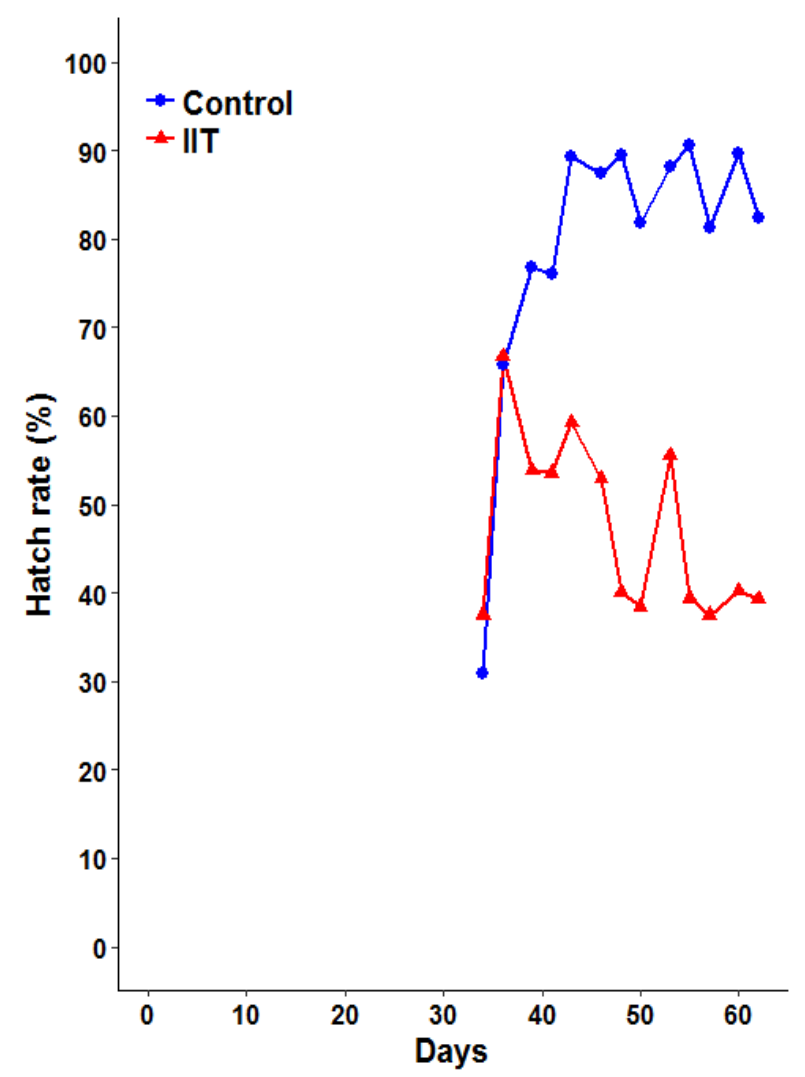

758 Fig. 6. Evaluation of the IIT effectiveness to limit D. suzukii population growth in a large 759 climatic chamber. A: number of eggs laid per $48 \mathrm{~h}$ at regular intervals. B: hatch rates at regular 760 intervals. 